\title{
Enhanced Anti-Cancer Effects of Conditioned Medium from Hypoxic Human Umbilical Cord-Derived Mesenchymal Stem Cells
}

\author{
Kyu-Hyun Han ${ }^{1}$, Ae-Kyeong Kim ${ }^{1}$, Gun-Jae Jeong ${ }^{1}$, Hye Ran Jeon ${ }^{1}$, Suk Ho Bhang ${ }^{2}$, Dong-ik Kim ${ }^{1}$ \\ ${ }^{1}$ Division of Vascular Surgery, Samsung Medical Center, Sungkyunkwan University School of Medicine, Seoul, Korea \\ ${ }^{2}$ Sungkyunkwan University School of Chemical Engineering, Suwon, Korea
}

Background and Objectives: There have been contradictory reports on the pro-cancer or anti-cancer effects of mesenchymal stem cells. In this study, we investigated whether conditioned medium (CM) from hypoxic human umbilical cord-derived mesenchymal stem cells (hUC-MSCs) (H-CM) showed enhanced anti-cancer effects compared with CM from normoxic hUC-MSCs (N-CM).

Methods and Results: Compared with N-CM, H-CM not only strongly reduced cell viability and increased apoptosis of human cervical cancer cells (HeLa cells), but also increased caspase-3/7 activity, decreased mitochondrial membrane potential (MMP), and induced cell cycle arrest. In contrast, cell viability, apoptosis, MMP, and cell cycle of human dermal fibroblast (hDFs) were not significantly changed by either CM whereas caspase-3/7 activity was decreased by H-CM. Protein antibody array showed that activin A, Beta IG-H3, TIMP-2, RET, and IGFBP-3 were upregulated in $\mathrm{H}-\mathrm{CM}$ compared with $\mathrm{N}-\mathrm{CM}$. Intracellular proteins that were upregulated by H-CM in HeLa cells were represented by apoptosis and cell cycle arrest terms of biological processes of Gene Ontology (GO), and by cell cycle of Kyoto Encyclopedia of Genes and Genomes (KEGG) pathways. In hDFs, negative regulation of apoptosis in biological process of GO and PI3K-Akt signaling pathway of KEGG pathways were represented.

Conclusions: H-CM showed enhanced anti-cancer effects on HeLa cells but did not influence cell viability or apoptosis of $\mathrm{hDF}$ and these different effects were supported by profiling of secretory proteins in both kinds of CM and intracellular signaling of HeLa cells and hDFs.

Keywords: Anti-cancer, Conditioned medium, Fibroblasts, Hypoxia, Mesenchymal stem cells

Received: January 2, 2019, Revised: March 5, 2019,

Accepted: March 6, 2019, Published online: April 30, 2019

Correspondence to Dong-ik Kim

Division of Vascular Surgery, Samsung Medical Center, Sunkyunkwan University School of Medicine, 81 Irwon-ro, Gangnamgu, Seoul 06351, Korea

Tel: +82-2-3410-3467, Fax: +82-2-3410-0040

E-mail: dikim@skku.edu

(a) This is an open-access article distributed under the terms of the Creative Commons Attribution Non-Commercial License (http://creativecommons.org/ licenses/by-nc/4.0/), which permits unrestricted non-commercial use, distribution, and reproduction in any medium, provided the original work is properly cited.

Copyright (c) 2019 by the Korean Society for Stem Cell Research

\section{Introduction}

The effects of mesenchymal stem cells on cancer cells in vitro or in vivo are controversial (1). Previous studies reported that human bone marrow mesenchymal stem cells promoted the growth of pancreatic cancer cells (2) and human adipose stem cells induced metastasis of breast cancer cells through secretory proteins (3). In contrast, human endometrial mesenchymal stem cells showed anti-cancer effects on human epithelial ovarian cancer cells in vitro and in vivo through paracrine factors (4), and human adipose mesenchymal stem cells suppressed proliferation of ovarian cancer cells through exosomal miRNA 
in vitro (5) and inhibited glioblastoma in the brain of xenograft (6).

Furthermore, there has been research to enhance the anti-cancer effects of mesenchymal stem cells, such as by overexpression of anti-cancer genes (7) or engineered delivery systems with an anti-cancer drug (8). However, these methods can reduce the viability of mesenchymal stem cells, modify endogenous genes, or exert toxicity on normal cells, and such negative effects limit the clinical application of cell therapy.

It has been reported that mesenchymal stem cells in hypoxic culture condition showed increased cell proliferation (9) and survival (10), maintenance of stemness (11) and reduced senescence (12) together with metabolic changes (13) and increased secretion of paracrine factors $(14,15)$. These paracrine factors showed enhanced beneficial effects on recovery from injury or disease in some experimental models (16-20). In addition, hypoxia conditioned human bone marrow mesenchymal stem cells promoted the growth, motility, and invasion of breast cancer cells through secretion of TGF- $\beta 1$ (21).

Based on this knowledge, we investigated whether conditioned medium from hypoxic human umbilical cord mesenchymal stem cells (hUC-MSCs) showed enhanced anti-cancer effects on HeLa cells compared with hDFs as non-cancer cells, and performed protein profiling analysis to associate intracellular signaling in both cell types with secretory proteins from hypoxic hUC-MSCs.

\section{Materials and Methods}

\section{Cell culture}

hUC-MSCs (PromoCell GmbH, Heidelberg, Germany) were cultured in Dulbecco's modified Eagle's medium (DMEM) supplemented with $10 \%$ fetal bovine serum (FBS) and $0.1 \%$ antibiotics (Gibco, Grand Island, NY, USA) at $37^{\circ} \mathrm{C}$ in a $5 \% \mathrm{CO}_{2}$ incubator (APM-30D; ASTEC, Fukuoka, Japan). From passage 4, hUC-MSCs were cultured at $21 \% \mathrm{O}_{2}$ as a normoxic condition or at $1 \% \mathrm{O}_{2}$ as a hypoxic condition. For analysis of proliferation of normoxic and hypoxic hUC-MSCs, $2 \times 10^{5}$ cells were cultured in $100-\mathrm{mm}$ culture plates for 5 days. When cell confluency reached $90 \%$, the cells were passaged using 0.25\% TrypsinEDTA (Gibco). At passage 6 of both kinds of cells, cell count was measured using Trypan blue $0.5 \%$ solution (Biowest, Riverside, MO, USA) and a hemocytometer. Cell viability was measured by addition of $100 \mu 1$ of CellTiter-Glo assay 2.0 reagents (Promega, Madison, WI, USA) to cells and incubation for 10 minutes. Luminescence as a value of cell viability was measured with GLO-
MAX Multi Detection System (Promega BioSystems Sunnyvale, CA, USA). HeLa cells (ATCC, Manassas, VA, USA) and hDFs (Gibco) were cultured in DMEM supplemented with $10 \%$ fetal bovine serum (FBS) and $0.1 \%$ antibiotics (Gibco) at $37^{\circ} \mathrm{C}$ in a $5 \% \mathrm{CO}_{2}$ incubator. Cells were used for experiments at passage 6 .

\section{Phenotyping of hUC-MSCs}

Normoxic and hypoxic hUC-MSCs at passage 6 were harvested, fixed with $\mathrm{BD}$ Cytofix Fixation Buffer (BD Biosciences, Piscataway, NJ, USA) for $10 \mathrm{~min}$ at room temperature and washed with Perm/Wash buffer (BD Biosciences). Cells were stained with V450 mouse anti-human CD31 (1:20), fluorescein isothiocyanate (FITC) mouse anti-human CD34 (1:20), phycoerythrin (PE)-Cy ${ }^{\mathrm{TM}} 7$ mouse anti-human CD44 (1:20), V500 mouse anti-human CD45 (1:20), PerCP-Cy ${ }^{\mathrm{TM}} 5.5$ mouse anti-human CD73 (1 : 20), PE mouse anti-human CD90 (1 : 20), APC mouse anti-human CD105 (1:20), or V450 mouse anti-human CD144 (1 : 20) (BD Biosciences) antibodies for 30 minutes at room temperature. Cells were washed with Stain Buffer (BD Biosciences), and analyzed with a Caliber flow cytometer (BD Biosciences) using CellQuest program (BD Biosciences) and Flowjo software (Treestar, San Carlos, CA, USA).

\section{Differentiation of hUC-MSCs}

Normoxic and hypoxic hUC-MSCs at passage $6\left(1.5 \times 10^{5}\right.$ cells) were seeded in 12-well culture plates with complete medium. After overnight incubation, cells were treated with adipogenic, osteogenic, and chondrogenic differentiation medium (CEFO, Seoul, Rep. of Korea). At day 28, adipogenic and osteogenic differentiation was analyzed with Oil Red $\mathrm{O}$ and Alizarin red (CEFO, Seoul, Rep. of Korea) according to the manufacturer's instructions. Quantification of differentiation was analyzed by measuring absorbance at $550 \mathrm{~nm}$ with a GLOMAX multi detection system. After 14 days of chondrogenic differentiation, cells were fixed with $4 \%$ formaldehyde (Sigma-Aldrich, St. Louis, MO, USA) for $30 \mathrm{~min}$, stained with $1 \%$ Alcian blue (Sigma-Aldrich) at room temperature overnight, and washed with $0.1 \mathrm{~N} \mathrm{HCl}$ (Sigma-Aldrich) and distilled water. To elute Alcian blue from cells, $8 \mathrm{M}$ guanidine $\mathrm{HCl}$ solution (Sigma-Aldrich) was added. After incubation at $4^{\circ} \mathrm{C}$ overnight, cell supernatant was transferred to 96-well plates and absorbance of released Alcian blue was measured at $600 \mathrm{~nm}$ with a GLOMAX multi detection system. Images of differentiated cells were acquired with a CKX41 inverted microscope (Olympus Corporation, Tokyo, Japan) and Infinity Analyze 6.1.0 software (Lumenera Corpora- 
tion, Ottawa, ON, Canada).

\section{Preparation of CM from hUC-MSCs}

Normoxic or hypoxic hUC-MSCs $\left(2 \times 10^{5}\right.$ cells $)$ were seeded in $100-\mathrm{mm}$ culture plates with complete medium. At day 5, when cell confluency reached approximately $90 \%$, cells were washed with $1 \times$ PBS and added to $6 \mathrm{ml}$ of DMEM medium without FBS or antibiotics. After incubation for 24 hours, CM from both cell lines was harvested and centrifuged at $300 \mathrm{~g}$ for 5 minutes. Supernatants were harvested and stored at $-80^{\circ} \mathrm{C}$. DMEM without FBS or antibiotics was used as control CM (C-CM). The $\mathrm{pH}$ of all $\mathrm{CM}$ was measured using a $\mathrm{PH}$ meter $\mathrm{PC}$ 440 (Corning, NY, USA).

\section{Cell viability assay}

HeLa cells or hDFs $\left(1 \times 10^{4}\right.$ cells $)$ were cultured overnight in 96-well white plates. The next day, the culture medium was removed and $100 \mu \mathrm{l}$ of C-CM, N-CM, or $\mathrm{H}-\mathrm{CM}$ was added to the cells. After 48 hours, $100 \mu \mathrm{l}$ of CellTiter-Glo assay 2.0 reagents (Promega, Madison, WI, USA) was added to the cells and incubated for 10 minutes. Luminescence was measured with GLOMAX Multi Detection System. Cell viability of normoxic and hypoxic hUCMSCs was analyzed with CellTiter-Glo assay 2.0 reagents after incubation of 1,000 cells in 96-well white plates for 5 days.

\section{Apoptosis assay}

HeLa cells or hDFs at passage $6\left(1.5 \times 10^{5}\right.$ cells $)$ were seeded in 6-well culture plates containing complete medium. After overnight incubation, cells were treated with $2 \mathrm{ml}$ of C-CM, N-CM and H-CM for 48 hours. Cells were harvested with $0.25 \%$ Trypsin-EDTA (Gibco, Grand Island, NY, USA) and stained with FITC Annexin V apoptosis Detection Kit I (BD Pharmingen, San Diego, CA, USA) according to the manufacturer's instructions. Stained cells were analyzed with a Caliber flow cytometer using CellQuest and data were analyzed using Flowjo software.

\section{Caspase $3 / 7$ activity assay}

HeLa cells or hDFs $\left(1 \times 10^{4}\right.$ cells $)$ were incubated overnight in 96-well white plates. The next day, the culture medium was removed and $100 \mu 1$ of C-CM, N-CM or $\mathrm{H}-\mathrm{CM}$ was added to the cells. After incubation for 12 or 24 hours, $100 \mu 1$ of Caspase-Glo 3/7 Assay reagent (Promega) was added to the cells and incubated for 1 hour. Luminescence as a value of caspase $3 / 7$ activity was measured with the GLOMAX Multi Detection System.

\section{Mitochondrial membrane potential assay}

HeLa cells or $\mathrm{hDF}$ ( $\left(1 \times 10^{4}\right.$ cells) were seeded in 96-well white plates and incubated overnight. The next day, the culture medium was removed and $100 \mu 1$ of control serum-free medium, N-CM, or H-CM was added to the cells. After incubation for 12 or 24 hours, the cells were assayed with the Orange Mitochondrial Membrane Potential Assay Kit (Abcam, Cambridge, UK) according to the manufacturer's instructions. Fluorescence $(\mathrm{Ex} / \mathrm{Em}=540 / 590$ $\mathrm{nm}$ ) as a value of mitochondrial membrane potential was measured with Mithras ${ }^{2}$ LB 943 Multimode Reader (Berthold Biotechnologies, Bad Wildad. Germany).

\section{Cell cycle assay}

HeLa cells or hDFs at passage 6 were seeded in 6-well culture plates containing complete medium at a concentration of $1.5 \times 10^{5}$ cells/well. After overnight incubation, cells were treated with $2 \mathrm{ml}$ of C-CM, N-CM, or H-CM for 24 hours. Cells were harvested with $0.25 \%$ TrypsinEDTA and fixed in $70 \%$ alcohol and $30 \%$ PBS at $4^{\circ} \mathrm{C}$ for 1 hour. Cells were stained with $20 \mu \mathrm{g} / \mathrm{ml}$ propidium iodide (PI; Abcam) and 1\% RNase A (QIAGEN, Valencia, $\mathrm{CA}$, USA) for 30 minutes at $37^{\circ} \mathrm{C}$. Stained cells were suspended in PBS and analyzed using a FACSVerse ${ }^{\mathrm{TM}}$ flow cytometer (BD Biosciences) and Flowjo software.

\section{Analysis of secretory protein by antibody-based protein array}

Secretory proteins in N-CM or H-CM were analyzed using a RayBio ${ }^{\circledR}$ Label-based (L-Series) Human L1000 Antibody Array (Raybiotech, Inc., Norcross, GA, USA) by E-biogen (Kyung Hee business center, Kyung Hee University, Seoul, Korea). Briefly, the Human L1000 Array slide was incubated with blocking solution at room temperature for 30 minutes and then with $400 \mu 1$ of sample for 2 hours at room temperature. The slide was then incubated with $1 \times$ biotin-conjugated anti-cytokine antibodies for 2 hours at room temperature before addition of $1 \times \mathrm{Cy} 3-$ conjugated Streptavidin stock solution and incubation for a further 2 hours at room temperature. After washing, the slide was scanned using a GenePix 4100A Scanner and quantified with GenePix 7.0 Software (Axon Instrument, USA). The data were analyzed using Genowiz 4.0TM (Ocimum Biosolutions, India) and proteins that were upregulated or downregulated in $\mathrm{H}-\mathrm{CM}$ compared with N-CM ( $>1.5$ fold) were annotated using UniProt DB and examined with GO and KEGG pathways of the Database for Annotation, Visualization and Integrated Discovery (DAVID) $(\mathrm{p}<0.01)$ 


\section{Signal pathway analysis by antibody-based protein array}

Intracellular proteins in HeLa cells or hDFs treated with $\mathrm{N}-\mathrm{CM}$ or $\mathrm{H}-\mathrm{CM}$ for $24 \mathrm{~h}$ were analyzed with a Signaling explorer antibody array (Full Moon BioSystems, Sunnyvale, CA, USA). Briefly, $50 \mu \mathrm{g}$ of protein sample was added to a microarray slide with 1,358 antibodies and the slide was treated with Cy3-streptavidin (GE Healthcare, Chalfont St. Giles, UK). The slide was washed with washing solution and rinsed with Milli-Q grade water. For data acquisition and analysis, the array slide was scanned with a GenePix 4100A scanner and the image was quantified with GenePix 7.0 Software. The data were analyzed using Genowiz 4.0TM) and protein information was annotated using the UniProt DB. Up- and down-regulated proteins $(>1.5$ fold $)$ were identified and analyzed with tools in DAVID $(\mathrm{p}<0.01)$.

\section{Statistical analysis}

Data analysis and statistics (t-tests) were conducted with GraphPad Prism version 6.01 (San Diego, CA, USA) and a $p$ value $<0.05$ was considered statistically significant.

\section{Results}

\section{$\mathrm{H}-\mathrm{CM}$ induced anti-cancer effects in HeLa cells}

Hypoxic hUC-MSCs showed increased cell proliferation and cell viability compared with normoxic hUC-MSCs (Supplementary Fig. S1A, S1B). Adipogenesis and chondrogenesis were significantly increased in hypoxic hUCMSCs compared with normoxic hUC-MSCs (Supplementary Fig. S1C, S1E), whereas osteogenesis was significantly decreased in hypoxic hUC-MSCs (Supplementary Fig. S1D). There was no change in phenotype markers in both cell types (Supplementary Fig. S1F, SG). Conditioned medium was prepared from both cell lines. There was no difference in $\mathrm{pH}$ between $\mathrm{CM}$ from normoxic hUC-MSCs (N-CM), hypoxic hUC-MSCs (H-CM), or DMEM without FBS or antibiotics as control (C-CM) (data not shown).

Treatment of HeLa cells with H-CM for 48 hours significantly decreased the viability of HeLa cells compared to treatment with $\mathrm{N}-\mathrm{CM}$ or C-CM (Fig. 1A). Consistent with cell viability, the proportion of cells that stained Annexin- $\mathrm{V}(-) / \mathrm{PI}(-)$ as live cells was significantly decreased, and the proportion that stained Annexin- $V(+) /$ $\mathrm{PI}(+)$ as late apoptotic cells was increased by treatment with H-CM (Fig. 1B, 1C). In addition, after treatment with $\mathrm{CM}$ for 12 or 24 hours, caspase-3/7 activity was dramatically increased (Fig. 1D) and mitochondrial mem- brane potential was decreased (Fig. 1E) in the presence of $\mathrm{H}-\mathrm{CM}$. Furthermore, treatment with $\mathrm{H}-\mathrm{CM}$ induced cell cycle arrest with increased G0/G1 phase and decreased S and G2/M phase at 24 hours compared to treatment with C-CM or N-CM (Fig. 1F, 1G).

\section{$\mathrm{H}-\mathrm{CM}$ does not influence cell viability of hDFs}

Next, we investigated the effect of $\mathrm{H}-\mathrm{CM}$ on hDFs as non-cancer cells. After treatment of hDFs with $\mathrm{CM}$ for 48 hours, there was no significant difference in cell viability and apoptosis between H-CM, N-CM, and C-CM (Fig. 2A $\sim \mathrm{C})$. Caspase- $3 / 7$ activity was significantly decreased at 24 hours after treatment with H-CM (Fig. 2D), whereas mitochondrial membrane potential was not significantly different among the CMs (Fig. 2E). Regarding cell cycle, in contrast to HeLa cells, the proportion of cells in $\mathrm{G} 0 / \mathrm{Gl}$ phase was decreased, and that of $\mathrm{S}$ and G2/M phase was increased, at 24 hours after treatment with $\mathrm{N}-\mathrm{CM}$ and H-CM compared to C-CM (Fig. 2F, 2G). However, there was no significant difference in cell cycle distribution only between treatment with N-CM and H-CM (Fig. 2F, 2G).

\section{Up- and down-regulated secretory proteins in $\mathrm{H}-\mathrm{CM}$ compared with $\mathrm{N}-\mathrm{CM}$}

To investigate anti-cancer-related factors in $\mathrm{H}-\mathrm{CM}$ we performed protein antibody array on secretory proteins in $\mathrm{H}-\mathrm{CM}$ and N-CM. Proteins with greater than 1.5-fold upregulation (red) or downregulation (green) in $\mathrm{H}-\mathrm{CM}$ compared with N-CM are shown in a cluster image (Fig. 3A, Supplementary Table S1). Among 10,000 proteins in the array, five proteins were upregulated and 33 proteins were downregulated in $\mathrm{H}-\mathrm{CM}$ ( $>1.5$ fold). Activin A (4.2656), Beta IG-H3 (3.6006), TIMP-2 (1.7886), RET (1.6723), and IGFBP-3 (1.5519) were upregulated more than 1.5-fold in H-CM compared with N-CM (Fig. 3A, Supplementary Table S1). Using GO analysis of DAVID, upregulated or downregulated proteins in H-CM ( $>1.5$ fold) compared with $\mathrm{N}-\mathrm{CM}$ were categorized as biological process or molecular function of GO terms ( $p<0.01$, Top 10 enriched) and the data were presented as the $-\log _{10} p$ value (Fig. $3 \mathrm{~B} \sim \mathrm{D}$, Supplementary Table S2, S3). Upregulated proteins were represented by biological processes of response to drug (3.0140), negative regulation of cell proliferation (2.7856), and positive regulation of extrinsic apoptotic signaling pathway in absence of ligand (2.6022) (Fig. 3B, Supplementary Table S2), but not by molecular function. Downregulated proteins were represented by biological processes of cell-cell signaling (6.5980), cytokine-mediated signaling pathway (4.0818), positive regulation of NF-kappaB transcription factor activity (4.0562), positive 

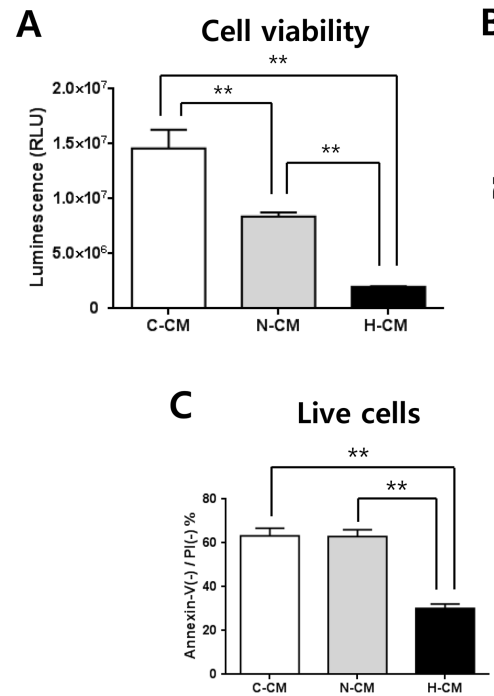

D

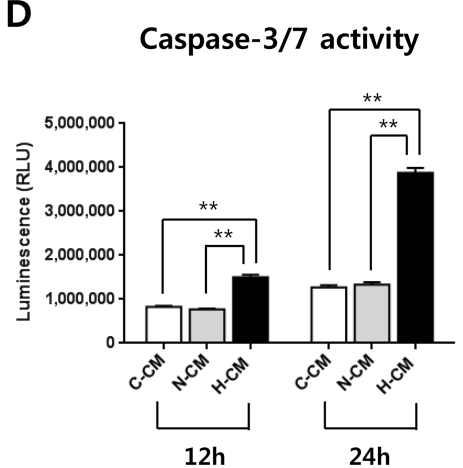

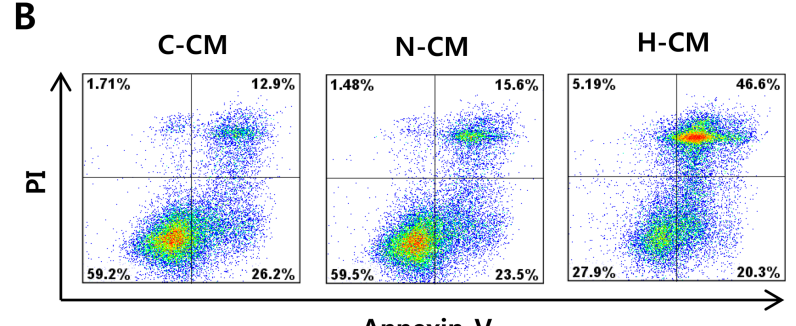

Annexin-V

Late apoptosis /dead cells

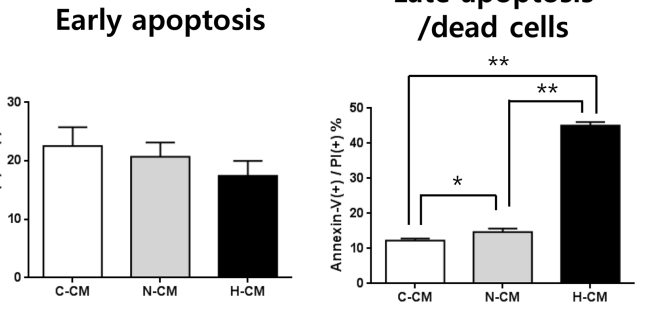

\section{E Mitochondrial membrane} potential
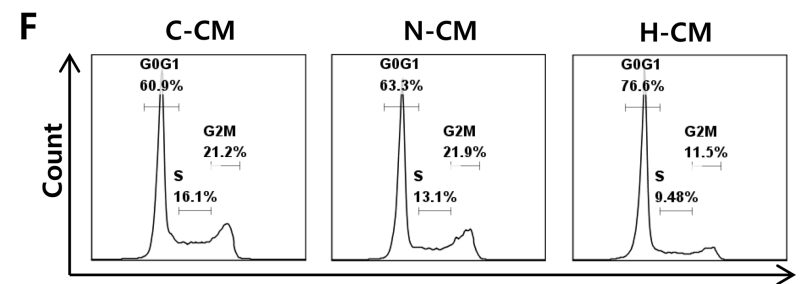

PI (DNA content)

G

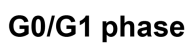

S phase

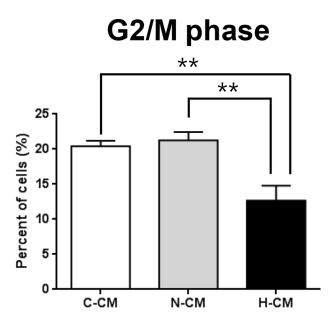

Fig. 1. H-CM induced enhanced anti-cancer effects in HeLa cells. Cell viability of HeLa cells was strongly decreased at 48 hours in the presence of $\mathrm{H}-\mathrm{CM}$ compared with $\mathrm{N}-\mathrm{CM}$ or control medium (A). The proportion of live cells $[\mathrm{Annexin}(-) / \mathrm{PI}(-)]$ was decreased, and that of late apoptotic cells $[$ Annexin $(+) / \mathrm{PI}(+)]$ at 48 hours was strongly increased, in the presence of $\mathrm{H}-\mathrm{CM}$ compared with $\mathrm{N}-\mathrm{CM}$ or control medium (B, C). Caspase-3/7 activity was strongly increased at 12 and 24 hours in the presence of $\mathrm{H}-\mathrm{CM}$ compared with $\mathrm{N}-\mathrm{CM}$ or control medium (D). Mitochondrial membrane potential was decreased at 12 and 24 hours in the presence of $\mathrm{H}-\mathrm{CM}$ compared with $\mathrm{N}-\mathrm{CM}$ or control medium (E). Cell cycle was arrested with an increase in G0/G1 and decrease in S and G2/M phases at 24 hours in the presence of $\mathrm{H}-\mathrm{CM}$ compared with $\mathrm{N}-\mathrm{CM}$ or control medium $(\mathrm{F}, \mathrm{G})$. Results are expressed as mean \pm S.E.M. from three independent experiments $\left(\mathrm{n}=3,{ }^{*} \mathrm{p}<0.05,{ }^{*} \mathrm{p}<0.01\right.$, t-test). C-CM: serum-free medium as control, N-CM: conditioned medium from normoxic hUC-MSCs, H-CM: conditioned medium from hypoxic hUC-MSCs. 


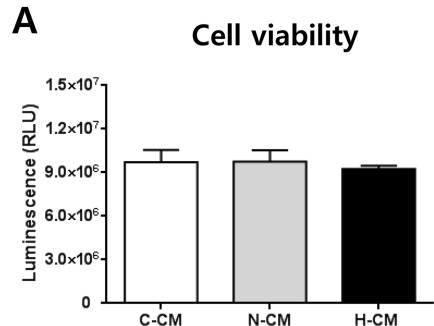

C

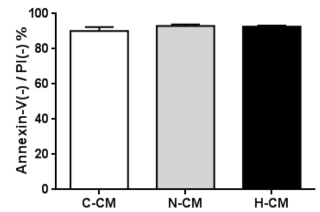

D

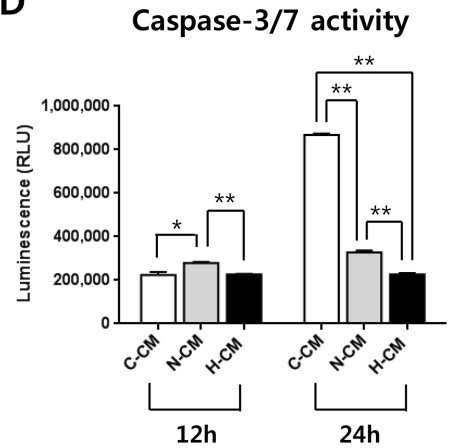

B

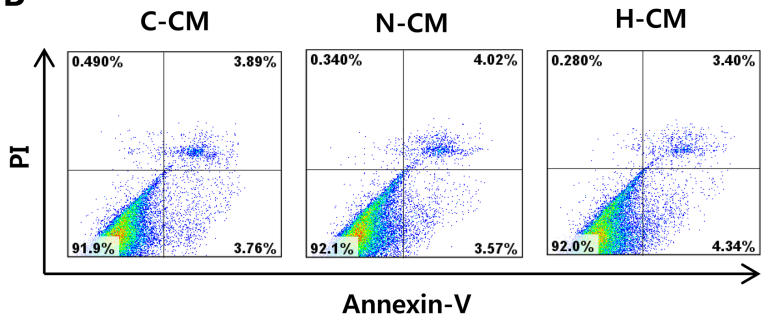

Late apoptosis /dead cells
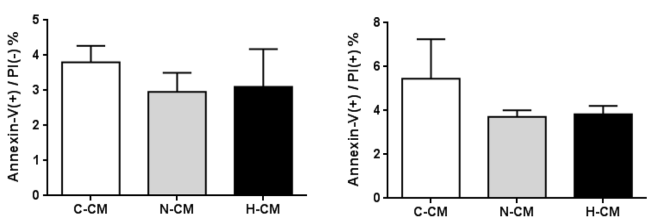

E Mitochondrial membrane potential

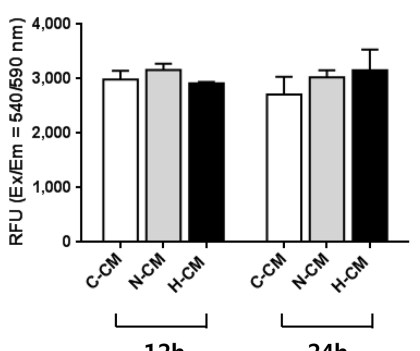

$\mathbf{F}$

C-CM

N-CM

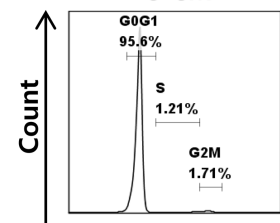

$\mathrm{H}-\mathrm{CM}$

${ }_{6061}{ }_{0061}$

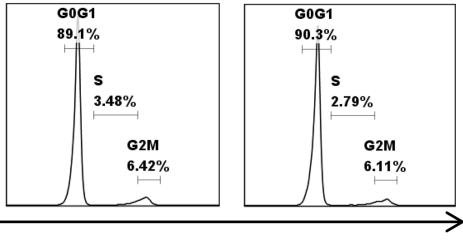

PI (DNA content)

G

G0/G1 phase

$S$ phase

G2/M phase
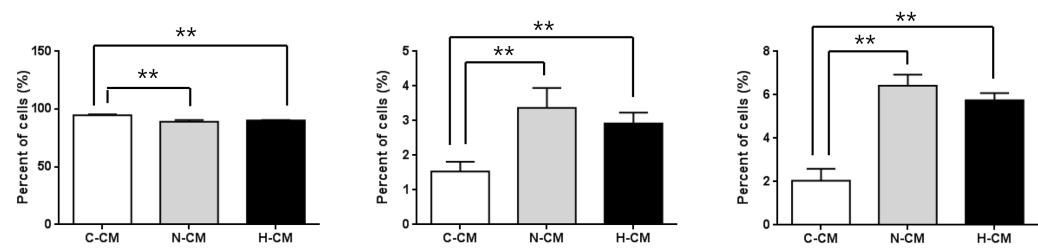

Fig. 2. H-CM did not affect cell viability of hDFs. Cell viability of hDFs at 48 hours was not significantly different in the presence of $\mathrm{H}-\mathrm{CM}, \mathrm{N}-\mathrm{CM}$, or C-CM (A). Apoptosis at 48 hours was not significantly different in the presence of $\mathrm{H}-\mathrm{CM}, \mathrm{N}-\mathrm{CM}$, or C-CM (B, C). Caspase-3/7 activity at 12 and 24 hours was decreased in the presence of $\mathrm{H}-\mathrm{CM}$ compared with N-CM or C-CM (D). Mitochondrial membrane potential was not changed at 12 and 24 hours in the presence of $\mathrm{H}-\mathrm{CM}$ compared with $\mathrm{N}-\mathrm{CM}$ or control medium (E). The proportion of cells in G0/G1 phase of the cell cycle was decreased, and S and G2/M phases were increased, after 24 hours in the presence of $\mathrm{H}-\mathrm{CM}$ or N-CM compared with control medium $(F, G)$. There was no significant difference in cell cycle distribution only between cells treated with $\mathrm{N}-\mathrm{CM}$ and $\mathrm{H}-\mathrm{CM},(\mathrm{F}, \mathrm{G})$. Results are expressed as mean \pm S.E.M. from three independent experiments $\left(\mathrm{n}=3,{ }^{*} \mathrm{p}<0.05,{ }^{* *} \mathrm{p}<0.01\right.$, t-test). C-CM, serum-free medium as control; $\mathrm{N}-\mathrm{CM}$, conditioned medium from normoxic hUC-MSCs; $\mathrm{H}-\mathrm{CM}$, conditioned medium from hypoxic hUC-MSCs. 
A

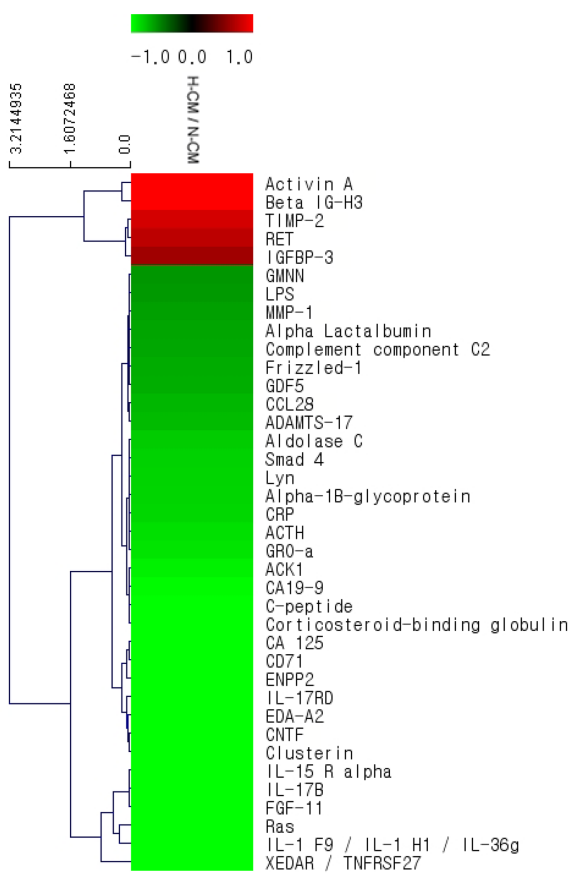

B

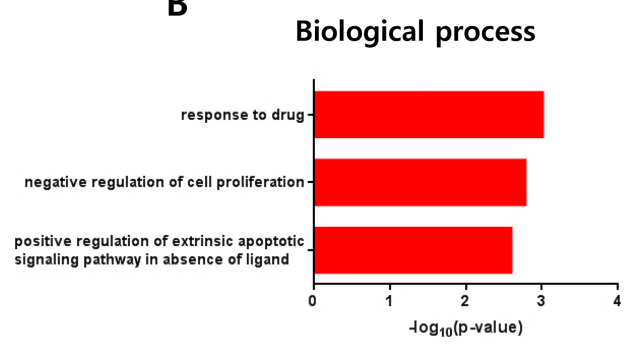

D

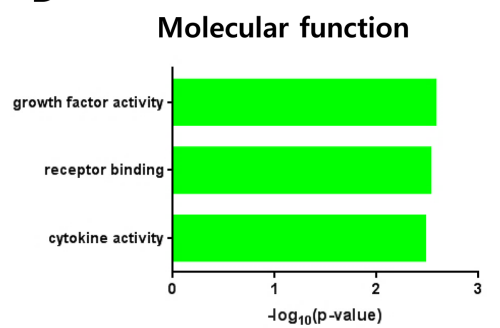

Fig. 3. Protein profiling of $\mathrm{H}-\mathrm{CM}$ compared with $\mathrm{N}-\mathrm{CM}$. Proteins that were upregulated (red) and downregulated (green) in $\mathrm{H}-\mathrm{CM}$ compared with $\mathrm{N}-\mathrm{CM}$ were analyzed using protein antibody array $(>1.5$ fold) (A). Biological process of $\mathrm{GO}$ analysis for upregulated proteins in $\mathrm{H}-\mathrm{CM}$ compared with N-CM (B). Biological process of GO analysis for downregulated proteins in $\mathrm{H}-\mathrm{CM}$ compared with N-CM (C). Molecular function of GO analysis for downregulated proteins in $\mathrm{H}-\mathrm{CM}$ compared with $\mathrm{N}-\mathrm{CM}$ (D). GO data were represented by the $-\log _{10} \mathrm{p}$ value $(\mathrm{p}<0.01)$ and top 10 enriched terms, and single sample analysis was performed.

regulation of gene expression (2.9381), signal transduction (2.4329), inflammatory response (2.3569), immune response (2.1968), and positive regulation of peptidyl-tyrosine phosphorylation (2.0279) (Fig. 3C, Supplementary Table S3), and by molecular functions of growth factor activity (2.5785), receptor binding (2.5297), and cytokine activity (2.4769) (Fig. 3D, Supplementary Table S3).

\section{$\mathrm{H}-\mathrm{CM}$ induced signal transduction in HeLa cells compared with $\mathrm{N}-\mathrm{CM}$}

Based on our results showing enhanced anti-cancer effects of H-CM compared with N-CM, we investigated in- tracellular signal transduction in HeLa cells induced by $\mathrm{H}-\mathrm{CM}$ and N-CM using protein antibody array. Among 1,358 proteins in the array, 53 proteins were upregulated (Fig. 4A, Supplementary Table S4) and 46 were downregulated (Fig. 4D, Supplementary Table S4) in HeLa cells $(>1.5$ fold) by treatment with $\mathrm{H}-\mathrm{CM}$ compared with $\mathrm{N}-\mathrm{CM}$. In GO analysis of upregulated proteins $(\mathrm{p}<0.01$, Top 10 enriched), for terms of biological process the highest $-\log _{10} \mathrm{p}$ value was for cell cycle arrest (3.1233) (Fig. 4B, Supplementary Table S5). In addition, apoptosis processes such as collagen catabolic process (3.0663), extracellular matrix disassembly (2.8497), negative regulation of 

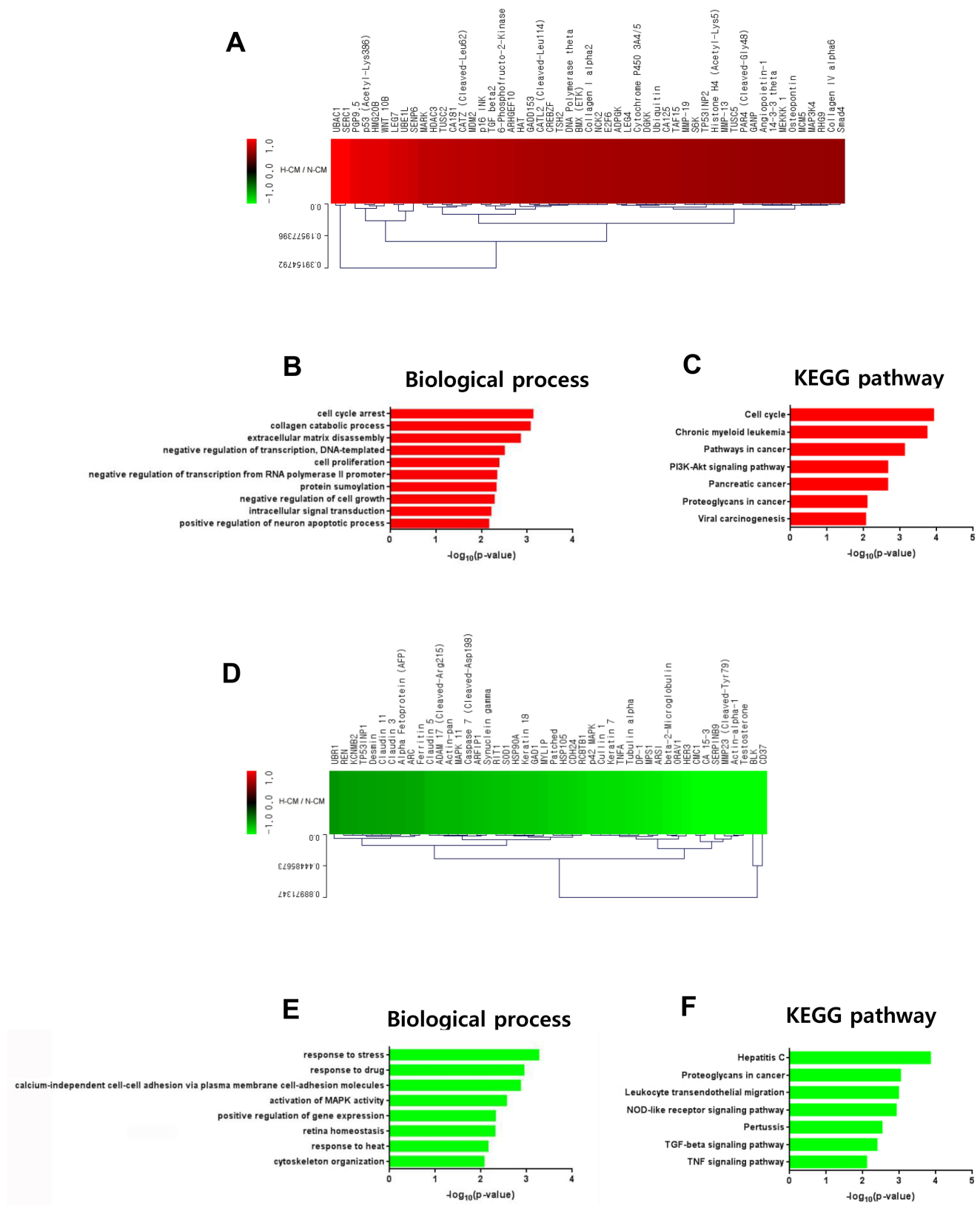

Fig. 4. Intracellular signaling induced by H-CM in HeLa cells. Upregulated proteins (red) involved in intracellular signaling of HeLa cells treated with $\mathrm{H}-\mathrm{CM}$ compared with N-CM according to protein antibody array ( $>1.5$ fold) (A). Biological process of GO analysis for upregulated intracellular proteins in HeLa cells treated with H-CM compared with N-CM (B). KEGG pathway analysis of upregulated intracellular proteins in HeLa cells treated with $\mathrm{H}-\mathrm{CM}$ compared with $\mathrm{N}-\mathrm{CM}(\mathrm{C})$. Downregulated proteins (green) involved in intracellular signaling of HeLa cells treated with $\mathrm{H}-\mathrm{CM}$ compared with $\mathrm{N}-\mathrm{CM}$ according to protein antibody array (>1.5 fold) (D). Biological process of GO analysis for downregulated intracellular proteins in HeLa cells treated with H-CM compared with N-CM (E). KEGG pathway analysis of downregulated intracellular proteins in HeLa cells treated with H-CM compared with N-CM (F). GO and KEGG pathway results were represented as the $-\log _{10} \mathrm{p}$ value $(\mathrm{p}<0.01)$ and top 10 enriched terms, and single sample analysis was performed.

cell growth (2.2772), and positive regulation of neuron apoptotic process (2.1562) were represented. Transcription activity, including negative regulation of transcription, DNA-templated (2.4999) and negative regulation of transcription from RNA polymerase II promoter (2.3345), cell proliferation (2.3812), protein sumoylation (2.3178), and intracellular signal transduction (2.2059) were also represented (Fig. 4B, Supplementary Table S5). In KEGG pathway analysis of upregulated proteins $(p<0.01$, Top 10 enriched), the highest $-\log _{10} \mathrm{p}$ value was for cell cycle 
(3.9167) (Fig. 4C, Supplementary Table S5). Cancer-related terms were chronic myeloid leukemia (3.7360), pathways in cancer (3.1183), pancreatic cancer (2.6575), viral carcinogenesis (2.0550), and proteoglycans in cancer (2.0922) (Fig. 4C, Supplementary Table S5). The PI3KAkt signaling pathway (2.6591) was also represented (Fig. 4C, Supplementary Table S5). In GO analysis of downregulated proteins, in terms of biological process $(\mathrm{p}<0.01$, Top 10 enriched), response activity including response to stress (3.2650), response to drug (2.9408), and response to heat (2.1542), calcium-independent cell-cell adhesion via plasma membrane cell-adhesion molecules (2.8648), activation of MAPK activity (2.5599), positive regulation of gene expression (2.3171), retina homeostasis (2.3086), and cytoskeleton organization (2.0659) were represented (Fig. 4E, Supplementary Table S6). In KEGG pathway analysis of downregulated proteins $(\mathrm{p}<0.01$, Top 10 enriched), NOD-like receptor signaling pathway (2.9139), TGF-beta

A

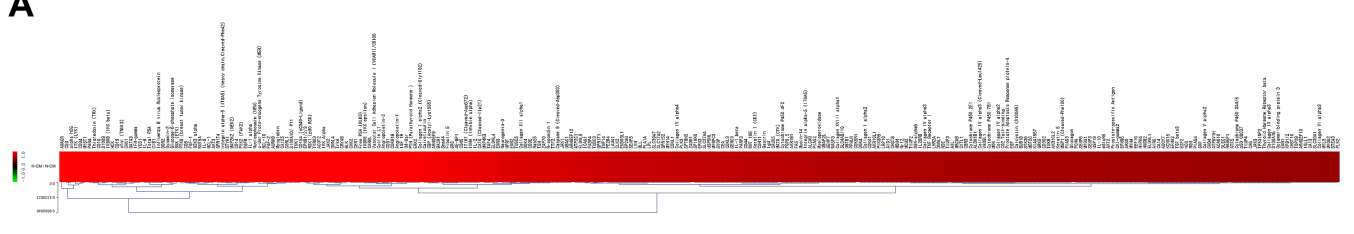

B
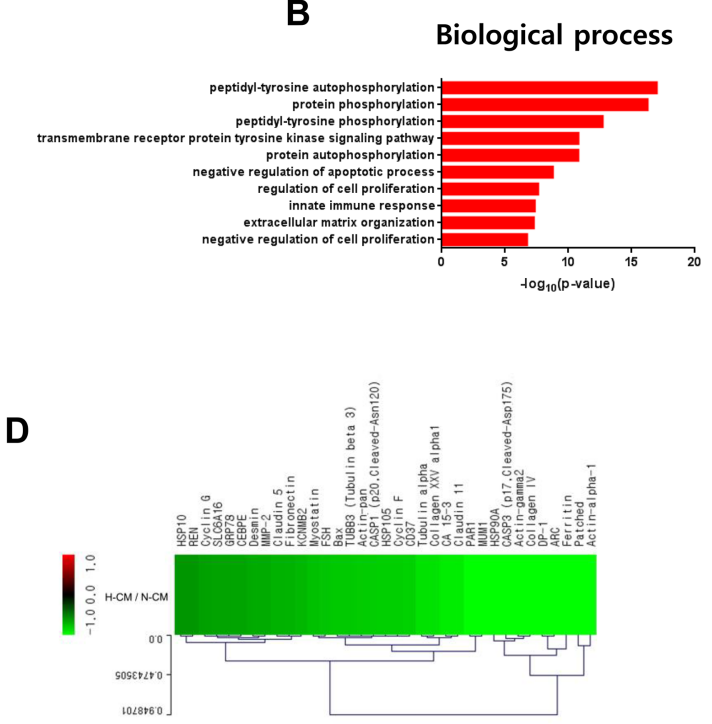

E

Biological process

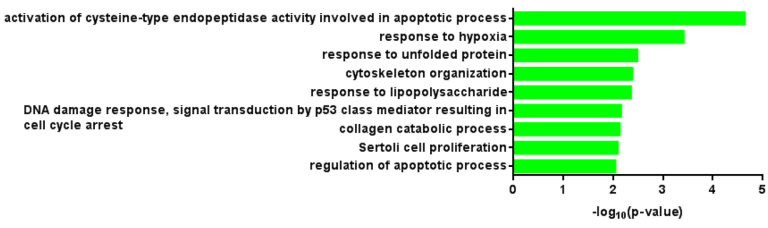

C

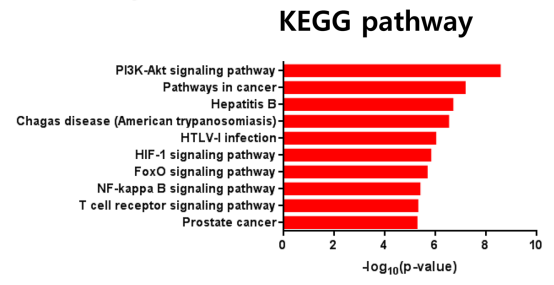

$\mathbf{F}$

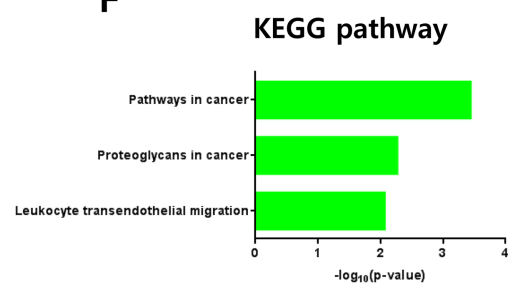

Fig. 5. Intracellular signaling induced by H-CM in hDFs. Upregulated proteins (red) involved in intracellular signaling of hDF cells treated with $\mathrm{H}-\mathrm{CM}$ compared with $\mathrm{N}-\mathrm{CM}$ according to protein antibody array ( $>1.5$ fold) (A). Biological process of GO analysis for upregulated intracellular proteins in hDFs treated with $\mathrm{H}-\mathrm{CM}$ compared with $\mathrm{N}-\mathrm{CM}(\mathrm{B})$. KEGG pathway analysis of upregulated intracellular proteins in hDFs treated with $\mathrm{H}-\mathrm{CM}$ compared with $\mathrm{N}-\mathrm{CM}(\mathrm{C})$. Downregulated proteins (green) involved in intracellular signaling of hDFs treated with $\mathrm{H}-\mathrm{CM}$ compared with $\mathrm{N}-\mathrm{CM}$ according to protein antibody array ( $>1.5$ fold) (D). Biological process of GO analysis for downregulated intracellular proteins in hDFs treated with $\mathrm{H}-\mathrm{CM}$ compared with $\mathrm{N}-\mathrm{CM}(\mathrm{E})$. KEGG pathway analysis of downregulated intracellular proteins in hDFs treated with $\mathrm{H}-\mathrm{CM}$ compared with $\mathrm{N}-\mathrm{CM}(\mathrm{F})$. GO and KEGG pathway results were represented by the $-\log _{10} \mathrm{p}$ value $(\mathrm{p}<0.01)$ and top 10 enriched terms, and single sample analysis was performed. 
signaling pathway (2.3865), and TNF signaling pathway (2.1049) were represented (Fig. 4F, Supplementary Table S6). In addition, hepatitis C (3.8517), proteoglycans in cancer (3.0326), leukocyte transendothelial migration (2.9802), and pertussis (2.5259) were also represented (Fig. $4 \mathrm{~F}$, Supplementary Table S6).

\section{Signal pathways in hDFs induced by H-CM compared with $\mathrm{N}-\mathrm{CM}$}

Based on our in vitro experimental data, we investigated induction of intracellular signal transduction in hDFs by H-CM compared with N-CM. Among 1,358 proteins in the protein antibody array, 276 proteins were upregulated (Fig. 5A, Supplementary Table S7) and 35 were downregulated (Fig. 5D, Supplementary Table S7) in hDFs (> 1.5 fold) by treatment with $\mathrm{H}-\mathrm{CM}$ compared with N-CM. In GO analysis of upregulated proteins, in terms of biological process $(\mathrm{p}<0.01$, Top 10 enriched), protein phosphorylation activity including peptidyl-tyrosine autophosphorylation (17.0101), protein phosphorylation (16.2848), peptidyl-tyrosine phosphorylation (12.7318), transmembrane receptor protein tyrosine kinase signaling pathway (10.8164), protein autophosphorylation (10.7996), and cell survival or proliferation activity including negative regulation of apoptotic process (8.7986), regulation of cell proliferation (7.6297), negative regulation of cell proliferation (6.7512), and innate immune response (7.3604), and extracellular matrix organization (7.2778) were represented (Fig 5B, Supplementary Table S8). In KEGG pathway analysis of upregulated proteins $(\mathrm{p}<0.01$, Top 10 enriched), the highest $-\log _{10} \mathrm{p}$ value was for PI3K-Akt signaling pathway (11.9321) (Fig 5C). For signal pathway activity, pathways in cancer (11.0537), HIF-1 signaling pathway (8.3131), FoxO signaling pathway (8.0767), NF-kappa $\mathrm{B}$ signaling pathway (7.1376), and $\mathrm{T}$ cell receptor signaling pathway (7.1131) were represented (Fig 5C, Supplementary Table S8). In addition, hepatitis B (9.2258), Chagas disease (American trypanosomiasis) (8.8724), HTLV-I infection (8.5068), and prostate cancer (7.0768) were also represented (Fig 5C, Supplementary Table S8). In GO analysis of downregulated proteins $(\mathrm{p}<0.01$, Top 10 enriched), for biological processes $(\mathrm{p}<0.01$, Top 10 enriched), the highest $-\log _{10} \mathrm{p}$ value was for activation of cysteine-type endopeptidase activity involved in apoptotic process (4.6386) (Fig. 5E, Supplementary Table S9). In addition, apoptosis or proliferation activity, such as DNA damage response, signal transduction by p53 class mediator resulting in cell cycle arrest (2.1565), Sertoli cell proliferation (2.0928), and regulation of apoptotic process (2.0450), response to hypoxia (3.4114), response to un- folded protein (2.4872), cytoskeleton organization (2.3806), response to lipopolysaccharide (2.3582), and collagen catabolic process (2.1298) were represented (Fig. 5E, Supplementary Table S9). In KEGG pathway analysis of downregulated proteins ( $\mathrm{p}<0.01$, Top 10 enriched), pathways in cancer (3.4454), proteoglycans in cancer (2.2720), and leukocyte transendothelial migration (2.0711) were represented (Fig. 5F, Supplementary Table S9).

\section{Discussion}

In this study, we demonstrated enhanced anti-cancer effects of hypoxic conditioned medium compared with normoxic conditioned medium from hUC-MSCs. Although $\mathrm{H}-\mathrm{CM}$ strongly induced apoptosis and cell cycle arrest of HeLa cancer cells followed by increased caspase-3/7 activity and decreased mitochondrial membrane potential, it did not affect the viability of hDFs as non-cancer cells; in fact, caspase-3/7 activity as an apoptosis marker in hDFs was decreased by treatment with H-CM compared with $\mathrm{N}-\mathrm{CM}$. This suggests that $\mathrm{H}-\mathrm{CM}$ would be an effective tool against cancer cells without affecting the viability of normal cells.

There have been controversial reports concerning whether mesenchymal stem cells show pro-cancer or anti-cancer effects (1). In addition, some research groups reported that hypoxic mesenchymal stem cells induced proliferation or enhanced the survival of cancer cells (21). In our experimental system, we treated HeLa cells with conditioned medium from hUC-MSCs in hypoxic or normoxic culture condition and with serum-free medium as control, and showed enhanced anti-cancer effects of H-CM. Activin A (4.2656), Beta IG-H3 (3.6006), TIMP-2 (1.7886), RET (1.6723), and IGFBP-3 (1.5519) were highly expressed in H-CM compared with N-CM. Previous studies reported that Activin A suppressed proliferation of HeLa cells (22), promoted metastatic growth of breast cancer (23), and stimulated cardiac fibroblasts (24). Beta IG-H3 was reported as a cancer suppressor or cancer promoter depending on the cancer microenvironment (25) and shown to promote adhesion and spreading of fibroblasts (26). TIMP-2 was reported to support survival of cancer cells (27), play a role in suppressing cancer promotion (28), and suppress collagen synthesis in fibroblasts (29). RET has been reported as a cancer suppressor gene (30) and oncogene (31). IGFBP-3 suppressed growth of melanoma (32) and modulated proliferation of fibroblasts (33). Even though some molecules appear to have a dual function in cancers based on previous reports, the proteins that are upregulated in H-CM may be critical factors for 
the enhanced anti-cancer effects of H-CM. As terms of biological process in $\mathrm{GO}$ analysis of intracellular proteins in HeLa cells that were upregulated in the presence of H-CM compared with N-CM, cell cycle arrest, collagen catabolic process, extracellular matrix disassembly, negative regulation of cell growth, and positive regulation of neural apoptosis were represented, and would be associated with the enhanced anti-cancer effects of H-CM. Regarding biological process terms of upregulated intracellular proteins in $\mathrm{hDF}$, negative regulation of apoptotic process, regulation of cell proliferation, and negative regulation of cell proliferation were represented. Furthermore, among downregulated proteins in hDFs, the most significant term of biological process was activation of cysteine endopeptidase activity involved in the apoptotic process (34). These results for intracellular signaling would be associated with the similar in vitro cell viability of hDFs treated with $\mathrm{H}-\mathrm{CM}$ or N-CM. In KEGG pathways of cancer cells treated with $\mathrm{H}-\mathrm{CM}$ compared with N-CM, the most significant pathway was cell cycle, which would be associated with the observed cell cycle arrest of cancer cells in the presence of H-CM. In particular, p53 (Acetyl-Lys386) was the most common molecule in biological process of GO analysis for proteins that were upregulated in HeLa cells by H-CM (Supplementary Table S5). In addition, p53 (AcetylLys386) showed the highest expression in cell cycle and PI3K-Akt signaling pathway of KEGG pathways for upregulated proteins in HeLa cells treated with H-CM (Supplementary Tables S4, S5). Previous studies identified p53 as a cancer suppressor (35) with a role in cell cycle arrest (36). Based on this knowledge, p53 appears to be the main player in signal transduction that mediates the enhanced anti-cancer effects of H-CM in HeLa cells. In KEGG pathways of downregulated proteins in HeLa cells treated with $\mathrm{H}-\mathrm{CM}, \mathrm{MARK} 11$ was included in all terms of KEGG pathway except TGF-beta signaling pathway (Supplementary Table S6). It has been reported that MAPK 11 is involved in cell survival (37). Enrichment of MARK11 in KEGG pathways of downregulated proteins of HeLa cells would be consistent with the reduced survival of HeLa cells exposed to $\mathrm{H}-\mathrm{CM}$ in the present study.

In the case of KEGG pathways in hDFs treated with $\mathrm{H}-\mathrm{CM}$, the PI3K-Akt signaling pathway was the most highly enriched. The PI3K-Akt signaling pathway is related to maintenance of survival of fibroblasts $(20,21)$. Furthermore, for downregulated proteins of hDFs, terms related to apoptosis or cell cycle arrest such as activation of cysteine-type endopeptidase activity involved in apoptotic process, DNA damage response, signal transduction by $\mathrm{p} 53$ class mediator resulting in cell cycle arrest, and regulation of apoptotic process were categorized among biological processes, and CASP3 (p17, Cleaved-Asp175) was included in terms such as Pathways in cancer and Proteoglycans in cancer in KEGG pathway analysis (Supplementary Table S9). These results would be related to maintained cell survival or decreased caspase-3/7 activity of hDFs treated with $\mathrm{H}-\mathrm{CM}$ compared to N-CM. These different effects of H-CM compared with $\mathrm{N}-\mathrm{CM}$ on cell viability, apoptosis, and intracellular signaling between cancer cells and human fibroblasts may be due to differences in expressed receptors and signal pathways between the two cell types.

Contrary to our results, a previous study reported that hypoxic conditioned medium from human bone marrow stem cells promoted progression of breast cancer cells through paracrine factors such as TGF- $\beta 1$ (21). These contradictory results may be due to differences in the sources of mesenchymal stem cells or cancer cells, experimental conditions for preparing CM such as hypoxic culture duration of cells, induction time of CM, and addition or non-addition of serum to prepare CM. A limitation of this research is that we only profiled secretory proteins in $\mathrm{H}-\mathrm{CM}$ compared with N-CM. According to a previous study (5), exosomal miRNA from mesenchymal stem cells may also mediate enhanced anti-cancer effects of H-CM and this warrants further study.

In conclusion, in the current study we demonstrated that conditioned medium from hypoxic human umbilical cord mesenchymal stem cells showed enhanced anti-cancer effects on HeLa cells without influencing the cell viability of non-cancer dermal fibroblasts, and profiled paracrine factors present in H-CM and the intracellular signaling pathways related to apoptosis of HeLa cells and survival of hDFs. Our results suggest the potential of culturing mesenchymal stem cells under hypoxic conditions in the development of alternative approaches to anti-cancer therapy.

\section{Acknowledgments}

This research was supported by the Bio \& Medical Technology Development Program of the National Research Foundation (NRF) funded by the Ministry of Science \& ICT (NRF-2018M3A9E2023255).

\section{Potential Conflict of Interest}

The authors have no conflicting financial interest.

\section{Supplementary Materials}

Supplementary data including nine tables and one fig- 
ure can be found with this article online at http://pdf. medrang.co.kr/paper/pdf/IJSC/IJSC-12-s19002.pdf.

\section{References}

1. Rhee KJ, Lee JI, Eom YW. Mesenchymal stem cell-mediated effects of tumor support or suppression. Int J Mol Sci 2015;16:30015-30033

2. Saito K, Sakaguchi M, Maruyama S, Iioka H, Putranto EW, Sumardika IW, Tomonobu N, Kawasaki T, Homma K, Kondo E. Stromal mesenchymal stem cells facilitate pancreatic cancer progression by regulating specific secretory molecules through mutual cellular interaction. J Cancer 2018;9:2916-2929

3. Gonzalez ME, Martin EE, Anwar T, Arellano-Garcia C, Medhora N, Lama A, Chen YC, Tanager KS, Yoon E, Kidwell KM, Ge C, Franceschi RT, Kleer CG. Mesenchymal stem cell-induced DDR2 mediates stromal-breast cancer interactions and metastasis growth. Cell Rep 2017;18: 1215-1228

4. Bu S, Wang Q, Zhang Q, Sun J, He B, Xiang C, Liu Z, Lai D. Human endometrial mesenchymal stem cells exhibit intrinsic anti-tumor properties on human epithelial ovarian cancer cells. Sci Rep 2016;6:37019

5. Reza AM, Choi YJ, Yasuda H, Kim JH. Human adipose mesenchymal stem cell-derived exosomal-miRNAs are critical factors for inducing anti-proliferation signalling to A2780 and SKOV-3 ovarian cancer cells. Sci Rep 2016;6: 38498

6. Pacioni S, D'Alessandris QG, Giannetti S, Morgante L, Coccè V, Bonomi A, Buccarelli $\mathrm{M}$, Pascucci L, Alessandri G, Pessina A, Ricci-Vitiani L, Falchetti ML, Pallini R. Human mesenchymal stromal cells inhibit tumor growth in orthotopic glioblastoma xenografts. Stem Cell Res Ther 2017;8:53

7. de Melo SM, Bittencourt S, Ferrazoli EG, da Silva CS, da Cunha FF, da Silva FH, Stilhano RS, Denapoli PM, Zanetti BF, Martin PK, Silva LM, dos Santos AA, Baptista LS, Longo BM, Han SW. The anti-tumor effects of adipose tissue mesenchymal stem cell transduced with HSV-Tk gene on U-87-driven brain tumor. PLoS One 2015;10:e128922

8. Yao S, Li X, Liu J, Sun Y, Wang Z, Jiang Y. Maximized nanodrug-loaded mesenchymal stem cells by a dual drug-loaded mode for the systemic treatment of metastatic lung cancer. Drug Deliv 2017;24:1372-1383

9. Li L, Jaiswal PK, Makhoul G, Jurakhan R, Selvasandran K, Ridwan K, Cecere R. Hypoxia modulates cell migration and proliferation in placenta-derived mesenchymal stem cells. J Thorac Cardiovasc Surg 2017;154:543-552.e3

10. Oh JS, Ha Y, An SS, Khan M, Pennant WA, Kim HJ, Yoon $\mathrm{DH}$, Lee M, Kim KN. Hypoxia-preconditioned adipose tissue-derived mesenchymal stem cell increase the survival and gene expression of engineered neural stem cells in a spinal cord injury model. Neurosci Lett 2010;472:215-219
11. Drela K, Sarnowska A, Siedlecka P, Szablowska-Gadomska I, Wielgos M, Jurga M, Lukomska B, Domanska-Janik K. Low oxygen atmosphere facilitates proliferation and maintains undifferentiated state of umbilical cord mesenchymal stem cells in an hypoxia inducible factor-dependent manner. Cytotherapy 2014;16:881-892

12. Tsai CC, Chen YJ, Yew TL, Chen LL, Wang JY, Chiu CH, Hung SC. Hypoxia inhibits senescence and maintains mesenchymal stem cell properties through down-regulation of E2A-p21 by HIF-TWIST. Blood 2011;117:459-469

13. Fujisawa K, Takami T, Okada S, Hara K, Matsumoto T, Yamamoto N, Yamasaki T, Sakaida I. Analysis of metabolomic changes in mesenchymal stem cells on treatment with desferrioxamine as a hypoxia mimetic compared with hypoxic conditions. Stem Cells 2018;36:1226-1236

14. Han KH, Kim AK, Kim MH, Kim DH, Go HN, Kang D, Chang JW, Choi SW, Kang KS, Kim DI. Protein profiling and angiogenic effect of hypoxia-cultured human umbilical cord blood-derived mesenchymal stem cells in hindlimb ischemia. Tissue Cell 2017;49:680-690

15. Song SW, Kim KE, Choi JW, Lee CY, Lee J, Seo HH, Lim KH, Lim S, Lee S, Kim SW, Hwang KC. Proteomic analysis and identification of paracrine factors in mesenchymal stem cell-conditioned media under hypoxia. Cell Physiol Biochem 2016;40:400-410

16. Chen L, Xu Y, Zhao J, Zhang Z, Yang R, Xie J, Liu X, Qi S. Conditioned medium from hypoxic bone marrow-derived mesenchymal stem cells enhances wound healing in mice. PLoS One 2014;9:e96161

17. Han KH, Kim AK, Kim MH, Kim DH, Go HN, Kim DI. Enhancement of angiogenic effects by hypoxia-preconditioned human umbilical cord-derived mesenchymal stem cells in a mouse model of hindlimb ischemia. Cell Biol Int 2016;40:27-35

18. Lan YW, Choo KB, Chen CM, Hung TH, Chen YB, Hsieh $\mathrm{CH}$, Kuo HP, Chong KY. Hypoxia-preconditioned mesenchymal stem cells attenuate bleomycin-induced pulmonary fibrosis. Stem Cell Res Ther 2015;6:97

19. Liu YY, Chiang CH, Hung SC, Chian CF, Tsai CL, Chen WC, Zhang H. Hypoxia-preconditioned mesenchymal stem cells ameliorate ischemia/reperfusion-induced lung injury. PLoS One 2017;12:e187637

20. Shin HS, Lee S, Kim YM, Lim JY. Hypoxia-activated adipose mesenchymal stem cells prevents irradiation-induced salivary hypofunction by enhanced paracrine effect through fibroblast growth factor 10. Stem Cells 2018;36:1020-1032

21. Hung SP, Yang MH, Tseng KF, Lee OK. Hypoxia-induced secretion of TGF- $\beta 1$ in mesenchymal stem cell promotes breast cancer cell progression. Cell Transplant 2013;22: 1869-1882

22. Katik I, Mackenzie-Kludas C, Nicholls C, Jiang FX, Zhou $\mathrm{S}, \mathrm{Li} \mathrm{H}$, Liu JP. Activin inhibits telomerase activity in cancer. Biochem Biophys Res Commun 2009;389:668-672

23. Bashir M, Damineni S, Mukherjee G, Kondaiah P. Activin-A signaling promotes epithelial-mesenchymal transition, invasion, and metastatic growth of breast cancer. 
NPJ Breast Cancer 2015;1:15007

24. Hu J, Wang X, Wei SM, Tang YH, Zhou Q, Huang CX. Activin A stimulates the proliferation and differentiation of cardiac fibroblasts via the ERK1/2 and p38-MAPK pathways. Eur J Pharmacol 2016;789:319-327

25. Ween MP, Oehler MK, Ricciardelli C. Transforming growth Factor-Beta-Induced Protein (TGFBI)/( $\beta$ ig-H3): a matrix protein with dual functions in ovarian cancer. Int J Mol Sci 2012;13:10461-10477

26. LeBaron RG, Bezverkov KI, Zimber MP, Pavelec R, Skonier J, Purchio AF. Beta IG-H3, a novel secretory protein inducible by transforming growth factor-beta, is present in normal skin and promotes the adhesion and spreading of dermal fibroblasts in vitro. J Invest Dermatol 1995; 104:844-849

27. Valacca C, Tassone E, Mignatti P. TIMP-2 interaction with MT1-MMP activates the AKT pathway and protects tumor cells from apoptosis. PLoS One 2015;10:e0136797

28. Chen Z, Zhu J, Zhu Y, Wang J. MicroRNA-616 promotes the progression of ovarian cancer by targeting TIMP2. Oncol Rep 2018;39:2960-2968

29. Dohi T, Miyake K, Aoki M, Ogawa R, Akaishi S, Shimada T, Okada T, Hyakusoku H. Tissue inhibitor of metalloproteinase-2 suppresses collagen synthesis in cultured keloid fibroblasts. Plast Reconstr Surg Glob Open 2015;3:e520

30. Luo Y, Tsuchiya KD, Il Park D, Fausel R, Kanngurn S, Welcsh P, Dzieciatkowski S, Wang J, Grady WM. RET is a potential tumor suppressor gene in colorectal cancer. Oncogene 2013;32:2037-2047
31. Kodama Y, Asai N, Kawai K, Jijiwa M, Murakumo Y, Ichihara M, Takahashi M. The RET proto-oncogene: a molecular therapeutic target in thyroid cancer. Cancer Sci 2005; 96:143-148

32. Naspi A, Panasiti V, Abbate F, Roberti V, Devirgiliis V, Curzio M, Borghi M, Lozupone F, Carotti S, Morini S, Gaudio E, Calvieri S, Londei P. Insulin-like-growth-factor-binding-protein-3 (IGFBP-3) contrasts melanoma progression in vitro and in vivo. PLoS One 2014;9:e98641

33. Izumi K, Kurosaka D, Iwata T, Oguchi Y, Tanaka Y, Mashima Y, Tsubota K. Involvement of insulin-like growth factor-I and insulin-like growth factor binding protein-3 in corneal fibroblasts during corneal wound healing. Invest Ophthalmol Vis Sci 2006;47:591-598

34. Schmid M, Simpson D, Gietl C. Programmed cell death in castor bean endosperm is associated with the accumulation and release of a cysteine endopeptidase from ricinosomes. Proc Natl Acad Sci U S A 1999;96:14159-14164

35. Yogosawa S, Yoshida K. Tumor suppressive role for kinases phosphorylating p53 in DNA damage-induced apoptosis. Cancer Sci 2018;109:3376-3382

36. Engeland K. Cell cycle arrest through indirect transcriptional repression by p53: I have a DREAM. Cell Death Differ 2018;25:114-132

37. Chun Yang X, Hui Zhao D, Bond Lau W, Qiang Liu K, Yu Tian J, Chao Cheng Z, Liang Ma X, Hua Liu J, Fan Q. IncRNA ENSMUST00000134285 increases MAPK11 activity, regulating aging-related myocardial apoptosis. J Gerontol A Biol Sci Med Sci 2018;73:1010-1017 